\title{
A Posteriori Error Analysis and Adaptive Methods for Partial Differential Equations
}

\author{
Zhiming Chen*
}

\begin{abstract}
The adaptive finite element method based on a posteriori error estimates provides a systematic way to refine or coarsen the meshes according to the local a posteriori error estimator on the elements. One of the remarkable properties of the method is that for appropriately designed adaptive finite element procedures, the meshes and the associated numerical complexity are quasi-optimal in the sense that the finite element discretization error is proportional to $N^{-1 / 2}$ in terms of the energy norm, where $N$ is the number of degrees of freedom of the underlying mesh. The purpose of this paper is to report some of the recent advances in the a posteriori error analysis and adaptive finite element methods for partial differential equations. Emphases will be paid on an adaptive perfectly matched layer technique for scattering problems and a sharp $L^{1}$ a posteriori error analysis for nonlinear convection-diffusion problems.
\end{abstract}

Mathematics Subject Classification (2000). Primary 65N15; Secondary 65N30.

Keywords. A posteriori error estimates, adaptivity, quasi-optimality

\section{Introduction}

The aim of the adaptive finite element method (AFEM) for solving partial differential equations is to find the finite element solution and the corresponding mesh with least possible number of elements with respect to discrete errors. The task to find the mesh with the desired property is highly nontrivial because the solution is a priori unknown. The basic idea of the seminal work [3] is to find the desired mesh under the principle of error equidistribution, that is, the discretization error should be approximately equal on each element. The error on the elements which is also unknown can, however, can be estimated by a posteriori error estimates. Today AFEM based on a posteriori error estimates attracts increasing interests and becomes one of the central themes of scientific computation. The purpose of this paper is to report some of the recent advances in the a posteriori error analysis and AFEM for partial differential equations.

A posteriori error estimates are computable quantities in terms of the discrete solution and data, which provide information for adaptive mesh refinement (and coarsening), error control, and equidistribution of the computational effort. We

\footnotetext{
${ }^{*}$ The author is grateful to the support of China National Basic Research Program under the grant 2005CB321701 and the China NSF under the grant 1002510 and 10428105.
} 
now describe briefly the basic idea of AFEM using the example of solving the Possion equation on a polygonal domain $\Omega$ in $\mathbf{R}^{2}$

$$
-\Delta u=f \text { in } \Omega, \quad u=0 \text { on } \partial \Omega .
$$

Here the source function $f$ is assumed to be in $L^{2}(\Omega)$. It is well-known that the solution of the problem (1.1) may be singular due to the reentrant corners of the domain in which case the standard finite element methods with uniform meshes are not efficient.

Let $\mathcal{M}_{h}$ be a regular triangulation of the domain $\Omega$ and $\mathcal{B}_{h}$ be the collection of all inter-element sides of $\mathcal{M}_{h}$. Denote by $u_{h}$ the piecewise linear conforming finite element solution over $\mathcal{M}_{h}$. For any inter-element side $e \in \mathcal{B}_{h}$, let $\Omega_{e}$ be the collection of two elements sharing $e$ and define the local error indicator $\eta_{e}$ as

$$
\eta_{e}^{2}:=\sum_{K \in \Omega_{e}}\left\|h_{K} f\right\|_{L^{2}(K)}^{2}+\left\|h_{e}^{1 / 2} J_{e}\right\|_{L^{2}(e)}^{2},
$$

where $h_{K}:=\operatorname{diam}(K), h_{e}:=\operatorname{diam}(e)$, and $J_{e}:=\llbracket \nabla u_{h} \rrbracket_{e} \cdot \nu$ stands for the jump of flux across side $e$ which is independent of the orientation of the unit normal $\nu$ to $e$. The following a posteriori error estimate is well-known [2]

$$
\left\|u-u_{h}\right\|_{H^{1}(\Omega)}^{2} \leq C \sum_{e \in \mathcal{B}_{h}} \eta_{e}^{2} .
$$

That $\eta_{e}$ really indicates the error is explained by the following lower bound [39].

$$
\eta_{e}^{2} \leq C \sum_{K \in \Omega_{e}}\left\|u-u_{h}\right\|_{L^{2}(K)}^{2}+C \sum_{K \in \Omega_{e}}\left\|h_{K}\left(f-f_{K}\right)\right\|_{L^{2}(K)}^{2},
$$

where $f_{K}=\frac{1}{|K|} \int_{K} f d x$.

Based on the local error indicator, the usual adaptive algorithm solving the elliptic problem (1.1) reads as follows

$$
\text { Solve } \rightarrow \text { Estimate } \rightarrow \text { Refine. }
$$

The important convergence property, which guarantees the iterative loop terminates in finite steps starting from an initial coarse mesh, is proved in [23, 30]. It is also observed (cf. e.g. [30]) that for appropriately designed adaptive finite element procedures, the meshes and the associated numerical complexity are quasi-optimal in the sense that

$$
\left\|\nabla\left(u-u_{h}\right)\right\|_{L^{2}(\Omega)} \approx C N^{-1 / 2}
$$

is valid asymptotically, where $N$ is the number of elements of the underlying finite element mesh. Since the nonlinear approximation theory [5] indicates that $N^{-1 / 2}$ is the highest attainable convergence order for approximating functions in $H^{1}(\Omega)$ in two space dimensions over a mesh with $N$ elements, one concludes that AFEM is an optimal discretization method for solving the elliptic problem (1.1). 
In section 2 we consider to use AFEM to solve the Helmholtz-type scattering problems with perfectly conducting boundary

$$
\begin{aligned}
& \Delta u+k^{2} u=0 \quad \text { in } \mathbf{R}^{2} \backslash \bar{D}, \\
& \frac{\partial u}{\partial \mathbf{n}}=-g \quad \text { on } \Gamma_{D}, \\
& \sqrt{r}\left(\frac{\partial u}{\partial r}-\mathbf{i} k u\right) \rightarrow 0 \quad \text { as } r=|x| \rightarrow \infty .
\end{aligned}
$$

Here $D \subset \mathbf{R}^{2}$ is a bounded domain with Lipschitz boundary $\Gamma_{D}, g \in H^{-1 / 2}\left(\Gamma_{D}\right)$ is determined by the incoming wave, and $\mathbf{n}$ is the unit outer normal to $\Gamma_{D}$. We assume the wave number $k \in \mathbb{R}$ is a constant. We study an adaptive perfectly matched layer (APML) technique to deal with the Sommerfeld radiation condition (1.3c) in which the PML parameters such as the thickness of the layer and the fictitious medium property are determined through sharp a posteriori error estimates. The APML technique combined with AFEM provides a complete numerical method for solving the scattering problem in the framework of finite element which has the nice property that the total computational costs are insensitive to the thickness of the PML absorbing layers. The quasi-optimality of underlying FEM meshes is also observed.

Things become much more complicated when applying AFEM to solve timedependent partial differential equations. One important question is if one should use the adaptive time marching (ATM) method in which variable timestep sizes (but constant at each time step) and variable space meshes at different time steps are assumed, or one should consider the the space-time adaptive method in which space-time domain is considered as a whole and AFEM is used without distinguishing the difference of time and space variables. Our recent studies in $[9,10,11]$ reveal that with sharp a posteriori error analysis and carefully designed adaptive algorithms, the ATM method also produces the very desirable quasi-optimal decay of the error with respect to the computational complexity

$$
\|u-U\|_{\Omega \times(0, T)} \leq C M^{-1 / 3}
$$

for a large class of convection-diffusion parabolic problems in two space dimensions using backward Euler scheme in time and conforming piecewise linear finite elements in space. Here $\|u-U\|_{\Omega \times(0, T)}$ is the energy norm of the error between the exact solution $u$ and the discrete solution $U$, and $M$ is the sum of the number of elements of the space meshes over all time steps. Thus if one takes the quasi-optimality of the computational complexity as the criterion to assess the adaptive methods, then the space-time adaptive method which is less studied in the literature will not have much advantage over the ATM method.

A posteriori error analysis for parabolic problems in the framework of ATM has been studied intensively in the literature. The main tool in deriving a posteriori error estimates in $[25,26,14,31,7]$ is the analysis of linear dual problems of the corresponding error equations. The derived a posteriori error estimates, however, 
depend on the $H^{2}$ regularity assumption on the underlying elliptic operator. Without using this regularity assumption, energy method is used in $[34,10]$ to derive an a posteriori error estimate for the total energy error of the approximate solution for linear heat equations. A lower bound for the local error is also derived for the associated a posteriori error indicator in [34, 9]. In [9] an adaptive algorithm is constructed which at each time step, is able to reduce the error indicators (and thus the error) below any given tolerance within finite number of iteration steps. Moreover, the adaptive algorithm is quasi-optimal in terms of energy norm. In [10] an quasi-optimal ATM method in terms of the energy norm is obtained for the linear convection-dominated diffusion problems based on $L^{1}$ a posteriori error estimates.

In section 3 we study the ATM method for the initial boundary value problems of nonlinear convection-diffusion equations of the form

$$
\frac{\partial u}{\partial t}+\operatorname{div} f(u)-\Delta A(u)=g .
$$

We derive sharp $L^{\infty}\left(L^{1}\right)$ a posteriori error estimates under the non-degeneracy assumption $A^{\prime}(s)>0$ for any $s \in \mathbf{R}$. The problem displays both parabolic and hyperbolic behavior in a way that depends on the solution itself. It is discretized implicitly in time via the method of characteristic and in space via continuous piecewise linear finite elements. The analysis is based on the Kružkov "doubling of variables" device and the recently introduced "boundary layer sequence" technique to derive the entropy error inequality on bounded domains. The derived a posteriori error estimate leads to a quasi-optimal adaptive method in terms of the norm $\|\cdot\|_{L^{1}}$ in (1.4).

\section{The APML technique for scattering problems}

In this section we consider the APML technique for the scattering problem (1.3a)(1.3c). Since [4] proposed a PML technique for solving the time dependent Maxwell equations, various constructions of PML absorbing layers have been proposed and studied in the literature $[38,37]$. Here we introduce the PML technique for (1.3a)(1.3c) following the method in [19].

Let $D$ be contained in the interior of the circle $B_{R}=\left\{x \in \mathbf{R}^{2}:|x|<R\right\}$. In the domain $\mathbf{R}^{2} \backslash \bar{B}_{R}$, the solution $u$ of (1.3a)-(1.3c) can be written under the polar coordinates as follows

$$
u(r, \theta)=\sum_{n \in \mathbb{Z}} \frac{H_{n}^{(1)}(k r)}{H_{n}^{(1)}(k R)} \hat{u}_{n} e^{\mathbf{i} n \theta}, \quad \hat{u}_{n}=\frac{1}{2 \pi} \int_{0}^{2 \pi} u(R, \theta) e^{-\mathbf{i} n \theta} d \theta .
$$

where $H_{n}^{(1)}$ is the Hankel function of the first kind and order $n$. The series in (2.1) converges uniformly for $r>R[20]$.

The basic idea of PML technique is to surround the fixed domain $\Omega_{R}=B_{R} \backslash \bar{D}$ with a PML layer of thickness $\rho-R$ and choose the fictitious medium property so 
that either the wave never reaches its external boundary or the amplitude of the reflected wave is so small that it does not essentially contaminate the solution in $\Omega_{R}$.

Let $\alpha=1+\mathbf{i} \sigma$ be the model medium property satisfying $\sigma \in C(\mathbf{R}), \sigma \geq 0$, and $\sigma=0$ for $r \leq R$. The most widely used model medium property $\sigma$ in the literature is the power function, that is,

$$
\sigma=\sigma_{0}\left(\frac{r-R}{\rho-R}\right)^{m}, \quad m \geq 1, \quad \sigma_{0}>0 \text { constant. }
$$

Denote by $\tilde{r}$ the complex radius defined by

$$
\tilde{r}=\tilde{r}(r)= \begin{cases}r & \text { if } r \leq R \\ \int_{0}^{r} \alpha(t) d t=r \beta(r) & \text { if } r \geq R\end{cases}
$$

Since $H_{n}^{(1)}(z) \sim \sqrt{\frac{2}{\pi z}} e^{\mathrm{i}\left(z-\frac{\pi}{2} n-\frac{\pi}{4}\right)}$ as $|z| \rightarrow \infty$, [19] obtained the PML equation by considering the following extension of $u$ in the exterior domain $\mathbf{R}^{2} \backslash \bar{B}_{R}$

$$
w(r, \theta)=\sum_{n \in \mathbb{Z}} \frac{H_{n}^{(1)}(k \tilde{r})}{H_{n}^{(1)}(k R)} \hat{u}_{n} e^{\mathrm{i} n \theta}, \quad \hat{u}_{n}=\frac{1}{2 \pi} \int_{0}^{2 \pi} u(R, \theta) e^{-\mathbf{i} n \theta} d \theta .
$$

It is easy to check that $w$ satisfies

$$
\nabla \cdot(A \nabla w)+\alpha \beta k^{2} w=0 \quad \text { in } \mathbf{R}^{2} \backslash \bar{B}_{R},
$$

where $A=A(x)$ is a matrix which satisfies, in polar coordinates,

$$
\nabla \cdot(A \nabla)=\frac{1}{r} \frac{\partial}{\partial r}\left(\frac{\beta r}{\alpha} \frac{\partial}{\partial r}\right)+\frac{\alpha}{\beta} \frac{1}{r^{2}} \frac{\partial^{2}}{\partial \theta^{2}} .
$$

The PML problem then becomes

$$
\begin{aligned}
& \nabla \cdot(A \nabla \hat{u})+\alpha \beta k^{2} \hat{u}=0 \quad \text { in } B_{\rho} \backslash \bar{D}, \\
& \frac{\partial \hat{u}}{\partial \mathbf{n}}=-g \text { on } \Gamma_{D}, \quad \hat{u}=0 \quad \text { on } \Gamma_{\rho} .
\end{aligned}
$$

It is proved in $[22,21]$ that the resultant PML solution converges exponentially to the solution of the original scattering problem as the thickness of the PML layer tends to infinity. We remark that in practical applications involving PML techniques, one cannot afford to use a very thick PML layer if uniform finite element meshes are used because it requires excessive grid points and hence more computer time and more storage. On the other hand, a thin PML layer requires a rapid variation of the artificial material property which deteriorates the accuracy if too coarse mesh is used in the PML layer.

The APML technique was first proposed in [16] for solving scattering by periodic structures (the grating problem) which uses a posteriori error estimates to determine the PML parameters such as the thickness and the medium property 
like $\sigma_{0}$ in the (2.2). For the scattering problem (1.3a)-(1.3c), the main difficulty of the analysis is that in contrast to the grating problems in which there are only finite number of outgoing modes [16], now there are infinite number of outgoing modes expressed in terms of Hankel functions. We overcome this difficulty by the by exploiting the following uniform estimate for the Hankel functions $H_{\nu}^{1}, \nu \in \mathbb{R}$.

Lemma 2.1. For any $\nu \in \mathbb{R}, z \in \mathbb{C}_{++}=\{z \in \mathbb{C}: \Im(z) \geq 0, \Re(z) \geq 0\}$, and $\Theta \in \mathbf{R}$ such that $0<\Theta \leq|z|$, we have

$$
\left|H_{\nu}^{(1)}(z)\right| \leq e^{-\Im(z)\left(1-\frac{\Theta^{2}}{|z|^{2}}\right)^{1 / 2}}\left|H_{\nu}^{(1)}(\Theta)\right| .
$$

The proof of the lemma which depends on the Macdonald formula for the modified Bessel functions can be found in [16]. Lemma 2.1 allows us to prove the exponentially decaying property of the PML solution without resorting to the integral equation technique in [22] or the representation formula in [21]. As a corollary of Lemma 2.1, we know that the function $w$ in (2.3) satisfies

$$
\|w\|_{H^{1 / 2}\left(\Gamma_{\rho}\right)} \leq e^{-k \Im(\tilde{\rho})\left(1-\frac{R^{2}}{|\tilde{\rho}|^{2}}\right)^{1 / 2}}\|u\|_{H^{1 / 2}\left(\Gamma_{R}\right)} .
$$

We remark that in [22], [21], it is required that the fictitious absorbing coefficient must be linear after certain distance away from the boundary where the PML layer is placed. We also remark that since $(2.5)$ is valid for all real order $\nu$, the results of [12] can be extended directly to study three dimensional Helmholtz-type scattering problems.

Let $\mathcal{M}_{h}$ be a regular triangulation of $B_{\rho} \backslash \bar{D}$ and $u_{h}$ be the finite element solution of the PML problem (2.4a)-(2.4b). Let $\mathcal{B}_{h}$ denote the set of all sides that do not lie on $\Gamma_{D}$ and $\Gamma_{\rho}^{h}$. For any $K \in \mathcal{M}_{h}$, we introduce the residual:

$$
R_{h}:=\nabla \cdot\left(\left.A \nabla u_{h}\right|_{K}\right)+\left.\alpha \beta k^{2} u_{h}\right|_{K} .
$$

For any interior side $e \in \mathcal{B}_{h}$ which is the common side of $K_{1}$ and $K_{2} \in \mathcal{M}_{h}$, we define the jump residual across $e$ :

$$
J_{e}:=\left(\left.A \nabla u_{h}\right|_{K_{1}}-\left.A \nabla u_{h}\right|_{K_{2}}\right) \cdot \nu_{e},
$$

using the convention that the unit normal vector $\nu_{e}$ to $e$ points from $K_{2}$ to $K_{1}$. If $e=\Gamma_{D} \cap \partial K$ for some element $K \in \mathcal{M}_{h}$, then we define the jump residual

$$
J_{e}:=2\left(\left.\nabla u_{h}\right|_{K} \cdot \mathbf{n}+g\right)
$$

For any $K \in \mathcal{M}_{h}$, denote by $\eta_{K}$ the local error estimator which is defined by

$$
\eta_{K}=\max _{x \in \tilde{K}} \omega(x) \cdot\left(\left\|h_{K} R_{h}\right\|_{L^{2}(K)}^{2}+\frac{1}{2} \sum_{e \subset \partial K} h_{e}\left\|J_{e}\right\|_{L^{2}(e)}^{2}\right)^{1 / 2},
$$

where $\tilde{K}$ is the union of all elements having nonempty intersection with $K$, and

$$
\omega(x)= \begin{cases}1 & \text { if } x \in \bar{B}_{R} \backslash \bar{D}, \\ \left|\alpha_{0} \alpha\right| e^{-k \Im(\tilde{r})\left(1-\frac{r^{2}}{|\tilde{r}|^{2}}\right)^{1 / 2}} & \text { if } x \in \bar{B}_{\rho} \backslash B_{R},\end{cases}
$$


Theorem 2.2. There exists a constant $C$ depending only on the minimum angle of the mesh $\mathcal{M}_{h}$ such that the following a posterior error estimate is valid

$$
\begin{aligned}
\left\|u-u_{h}\right\|_{H^{1}\left(\Omega_{R}\right) \leq} & C \Lambda(k R)^{1 / 2}(1+k R)\left(\sum_{K \in \mathcal{M}_{h}} \eta_{K}^{2}\right)^{1 / 2} \\
& +C(1+k R)^{2}\left|\alpha_{0}\right|^{2} e^{-k \Im(\tilde{\rho})\left(1-\frac{R^{2}}{|\tilde{\rho}|^{2}}\right)^{1 / 2}}\left\|u_{h}\right\|_{H^{1 / 2}\left(\Gamma_{R}\right)} .
\end{aligned}
$$

Here $\Lambda(k R)=\max \left(1, \frac{\left|H_{0}^{(1) \prime}(k R)\right|}{\left|H_{0}^{(1)}(k R)\right|}\right)$.

From Theorem 3.1 we know that the a posteriori error estimate consists of two parts: the PML error and the finite element discretization error. An adaptive algorithm is developed in [12] which uses the a posteriori error estimate to determine the PML parameters. We first choose $\rho$ and $\sigma_{0}$ such that the exponentially decaying factor

$$
\hat{\omega}=e^{-k \Im(\tilde{\rho})\left(1-\frac{R^{2}}{|\hat{\rho}|^{2}}\right)^{1 / 2}} \leq 10^{-8},
$$

which makes the PML error negligible compared with the finite element discretization errors. Once the PML region and the medium property are fixed, we use the standard finite element adaptive strategy to modify the mesh according to the a posteriori error estimate. The extensive numerical experiments reported in [12] show the competitive behavior of the proposed adaptive method. In particular, the quasi-optimality of meshes is observed and the adaptive algorithm is robust with respect to the choice of the thickness of PML layer: the far fields of the scattering solutions are insensitive to the choices of the PML parameters.

\section{The ATM method for nonlinear convection diffusion problems}

Let $\Omega$ is a bounded domain in $\mathbf{R}^{d}(d=1,2,3)$ with Lipschitz boundary and $T>0$. We consider the following nonlinear convection-diffusion equation

$$
\frac{\partial u}{\partial t}+\operatorname{div} f(u)-\Delta A(u)=g \quad \text { in } Q
$$

with the initial and boundary conditions

$$
\left.u\right|_{t=0}=u_{0},\left.\quad u\right|_{\partial \Omega \times(0, T)}=0 .
$$

Here $u=u(x, t) \in \mathbf{R}$, with $(x, t) \in Q=\Omega \times(0, T)$. We assume that the function $f$ : $\mathbf{R} \rightarrow \mathbf{R}^{d}$ is locally Lipschitz continuous, the function $A: \mathbf{R} \rightarrow \mathbf{R}$ is nondecreasing and locally Lipschitz continuous, $g \in L^{\infty}(Q)$ and $u_{0} \in L^{\infty}(\Omega)$.

Problems of the type (3.1) model a wide variety of physical phenomena including porous media flow, flow of glaciers and sedimentation processes, or flow 
transport through unsaturated porous media which is governed by the so-called Richards equation. For the Richards equation, the existence of weak solutions is considered in [1] and the uniqueness of weak solutions is proved in [33] based on the Kružkov "doubling of variables" technique. Entropy solutions for (3.1) are studied in [6, 29].

The discretization of (3.1) is based on combining continuous piecewise linear finite elements in space with the characteristic finite difference in time. The method of characteristic originally proposed in $[24,35]$ is widely used to solve convectiondiffusion problems in finite element community (cf. e.g. [26, 14]). Given $U_{h}^{n-1}$ as the finite element approximation of the solution at time $t^{n-1}$, let $\tau_{n}$ and $V_{0}^{n} \subset$ $H_{0}^{1}(\Omega)$ be the time step and the conforming linear finite element space at the $n$th time step, then our discrete scheme reads as following: find $U_{h}^{n} \in V_{0}^{n}$ such that

$$
\left\langle\frac{U_{h}^{n}-\bar{U}_{h}^{n-1}}{\tau_{n}}, v\right\rangle+\left\langle\nabla A\left(U_{h}^{n}\right), \nabla v\right\rangle=\left\langle\bar{g}^{n}, v\right\rangle \quad \forall v \in V_{0}^{n},
$$

where $\bar{g}^{n}=\tau_{n}^{-1} \int_{t^{n-1}}^{t^{n}} g(x, t) d t, \bar{U}_{h}^{n-1}(x)=U_{h}^{n-1}\left(\tilde{X}\left(t^{n-1}\right)\right)$, and the approximate characteristic $\tilde{X}(t)$ is defined by

$$
d \tilde{X} / d t=f^{\prime}\left(U_{h}^{n-1}(\tilde{X}(t))\right), \quad \tilde{X}\left(t^{n}\right)=x .
$$

The well-known Kružkov "doubling of variables" technique originally appeared in [28] plays a decisive role in the error estimation (both a posteriori and a priori) for numerical schemes solving the Cauchy problems of nonlinear conservation laws (see e.g. [17, 18, 27] and the reference therein). It is also used recently in [32] for the implicit vortex centered finite volume discretization of the Cauchy problems of (3.1) for general non-negative $A^{\prime}(s) \geq 0$ for all $s \in \mathbf{R}$. The common feature of these studies is that the derived error indicators are of the order $\sqrt{h}$ in the region where the solution is smooth, where $h$ is the local mesh size. We remark that in the region where the diffusion is dominant, the error indicators developed for the parabolic equations (cf. e.g. $[34,9]$ ) are of order $h$. Thus the degeneration of the order of the error indicators used in [32] may cause over-refinements for the solution of (3.1) in the region where the diffusion is dominant.

The basic assumption in this paper is that the diffusion is positive

$$
A^{\prime}(s)>0, \quad \forall s \in \mathbf{R} .
$$

This assumption includes the Richards equation and the viscosity regularization of degenerate parabolic equations, for example, the regularized continuous casting problem which is considered in [14]. The novelty of our analysis with respect to the analysis for nonlinear conservation laws in $[17,18,27]$ or nonlinear degenerate parabolic equations in [32] lies in the following aspects. Firstly, only Cauchy problems are considered in $[17,18,27,32]$. The difficulty to include boundary condition is essential. Here we use the recently introduced technique of "boundary layer sequence" in [29] to overcome the difficulty. The technique of "boundary layer sequence" allows us to truncate the standard Kružkov test function (see Definition 
3.4 below) to obtain the admissible test function in the entropy error identity. Secondly, the nature of the estimators are different: our estimators emphasize the diffusion effect of the problem which requires the assumption $A^{\prime}(s)>0$ for any $s \in \mathbf{R}$; the estimates in [32] are valid for any nonlinear function $A$ such that $A^{\prime}(s) \geq 0$. The nice consequence of the analysis is that our a posteriori error estimates are able to recover the standard sharp a posteriori error estimators in the literature derived for parabolic problem with diffusion coefficients bounded uniformly away from zero.

Now we elaborate the main steps to derive sharp $L^{1}$ a posteriori error estimate for the discrete scheme (3.3) based on the Kružkov "doubling of variables" device. By testing $(3.1)$ with any function $\varphi \in L^{2}\left(0, T ; H_{0}^{1}(\Omega)\right)$ such that $\phi(\cdot, 0)=\phi(\cdot, T)=$ 0 , we have

$$
\int_{0}^{T}\left\langle\partial_{t} u, \varphi\right\rangle d t+\int_{Q}(-f(u)+\nabla A(u)) \cdot \nabla \varphi d x d t=\int_{Q} g \varphi d x d t .
$$

For any $\varepsilon>0$, let

$$
H_{\varepsilon}(z)=\operatorname{sgn}(z) \min (1,|z| / \varepsilon)
$$

be the regularization of the sign function $\operatorname{sgn}(z)$. For any $k \in \mathbf{R}$, define the entropy pair $\left(U_{\varepsilon}, F_{\varepsilon}\right)$ by

$$
U_{\varepsilon}(z, k)=\int_{k}^{z} H_{\varepsilon}(A(r)-A(k)) d r, \quad F_{\varepsilon}(z, k)=\int_{k}^{z} H_{\varepsilon}(A(r)-A(k)) f^{\prime}(r) d r .
$$

The following result is well-known (cf. e.g. [6, 29]) by taking $\varphi=H_{\varepsilon}(A(u)-A(k)) \phi$ in (3.4).

Lemma 3.1. For any $\phi \in L^{2}\left(0, T ; H_{0}^{1}(\Omega)\right)$ such that $\phi(\cdot, 0)=\phi(\cdot, T)=0$, and any $k \in \mathbf{R}$, we have

$$
\begin{gathered}
-\int_{Q} U_{\varepsilon}(u, k) \partial_{t} \phi-\int_{Q} F_{\varepsilon}(u, k) \cdot \nabla \phi+\int_{Q} H_{\varepsilon}(A(u)-A(k)) \nabla A(u) \cdot \nabla \phi \\
+\int_{Q} H_{\varepsilon}^{\prime}(A(u)-A(k))|\nabla A(u)|^{2} \phi=\int_{Q} g H_{\varepsilon}(A(u)-A(k)) \phi .
\end{gathered}
$$

Let $\left(H^{1}(\Omega)\right)^{\prime}$ be the dual space of $H^{1}(\Omega)$, we define the discrete residual $\mathcal{R} \in$ $L^{2}\left(0, T ;\left(H^{1}(\Omega)\right)^{\prime}\right)$ through the following relation, for any $\varphi \in H^{1}(\Omega)$,

$$
\left\langle\partial_{t} U_{h}, \varphi\right\rangle-\left\langle f\left(U_{h}\right), \nabla \varphi\right\rangle+\left\langle\nabla A\left(U_{h}\right), \nabla \varphi\right\rangle=\langle g, \varphi\rangle-\langle\mathcal{R}, \varphi\rangle .
$$

For any $k^{\prime} \in \mathbf{R}$, by taking $\varphi=H_{\varepsilon}\left(A\left(U_{h}\right)-A\left(k^{\prime}\right)\right) \phi$ in (3.6), we have the following result. 
Lemma 3.2. For any $\phi \in L^{2}\left(0, T ; H_{0}^{1}(\Omega)\right)$ such that $\phi(\cdot, 0)=\phi(\cdot, T)=0$, and any $k^{\prime} \in \mathbf{R}$, we have

$$
\begin{aligned}
& -\int_{Q} U_{\varepsilon}\left(U_{h}, k^{\prime}\right) \partial_{t} \phi-\int_{Q} F_{\varepsilon}\left(U_{h}, k^{\prime}\right) \cdot \nabla \phi+\int_{Q} H_{\varepsilon}\left(A\left(U_{h}\right)-A\left(k^{\prime}\right)\right) \nabla A(u) \cdot \nabla \phi \\
& \quad+\int_{Q} H_{\varepsilon}^{\prime}\left(A\left(U_{h}\right)-A\left(k^{\prime}\right)\right)\left|\nabla A\left(U_{h}\right)\right|^{2} \phi \\
& \quad=\int_{Q} g H_{\varepsilon}\left(A\left(U_{h}\right)-A\left(k^{\prime}\right)\right) \phi-\int_{0}^{T}\left\langle\mathcal{R}, H_{\varepsilon}\left(A\left(U_{h}\right)-A\left(k^{\prime}\right)\right) \phi\right\rangle
\end{aligned}
$$

Now we are going to apply the Kružkov "doubling of variables" technique and will always write $u=u(y, s), U_{h}=U_{h}(x, t)$, unless otherwise stated. By taking $k=U_{h}(x, t)$ in $(3.5)$ and $k^{\prime}=u(y, s)$ in (3.7), we have the following entropy error identity.

Lemma 3.3. Let $\phi=\phi(x, t ; y, s)$ be non-negative function such that

$$
\begin{aligned}
& (x, t) \mapsto \phi(x, t ; y, s) \in C_{c}^{\infty}(Q) \text { for every }(y, s) \in Q, \\
& (y, s) \mapsto \phi(x, t ; y, s) \in C_{c}^{\infty}(Q) \text { for every }(x, t) \in Q .
\end{aligned}
$$

Then we have

$$
\begin{aligned}
& -\int_{Q \times Q} U_{\varepsilon}\left(u, U_{h}\right)\left(\partial_{t} \phi+\partial_{s} \phi\right)-\int_{Q \times Q} F_{\varepsilon}\left(u, U_{h}\right)\left(\nabla_{x} \phi+\nabla_{y} \phi\right) \\
& +\int_{Q \times Q} H_{\varepsilon}\left(A(u)-A\left(U_{h}\right)\right) \nabla_{y} A(u) \cdot\left(\nabla_{x} \phi+\nabla_{y} \phi\right) \\
& +\int_{Q \times Q} H_{\varepsilon}\left(A\left(U_{h}\right)-A(u)\right) \nabla_{x} A\left(U_{h}\right) \cdot\left(\nabla_{x} \phi+\nabla_{y} \phi\right) \\
& +\int_{Q \times Q} H_{\varepsilon}^{\prime}\left(A(u)-A\left(U_{h}\right)\right)\left|\nabla_{x} A\left(U_{h}\right)-\nabla_{y} A(u)\right|^{2} \phi \\
= & \left.-\int_{Q \times Q} \partial_{t}\left[U_{\varepsilon}\left(U_{h}, u\right)-U_{\varepsilon}\left(u, U_{h}\right)\right)\right] \phi \\
& \left.-\int_{Q \times Q} \nabla_{x}\left[F_{\varepsilon}\left(U_{h}, u\right)-F_{\varepsilon}\left(u, U_{h}\right)\right)\right] \phi \\
& -\int_{Q_{(y, s)}} \int_{0}^{T}\left\langle\mathcal{R}, H_{\varepsilon}\left(A\left(U_{h}\right)-A(u)\right) \phi\right\rangle d t .
\end{aligned}
$$

The next objective is to remove the restriction that the test functions in the entropy error identity (3.8) must have vanishing trace. This is achieved by using the technique of boundary layer sequence introduced in [29]. For any $\delta>0$, the boundary layer sequence $\zeta_{\delta}$ is defined as the solution of the elliptic problem

$$
-\delta^{2} \Delta \zeta_{\delta}+\zeta_{\delta}=1 \quad \text { in } \Omega, \quad \zeta_{\delta}=0 \quad \text { on } \partial \Omega .
$$

We specify now the choice of the test function $\phi$ in the entropy error identity (3.8), which is similar to that used in [29]. 
Definition 3.4. Let

$$
\phi(x, t, y, s)=\zeta_{\delta}(x) \zeta_{\eta}(y) \xi(x, t, y, s) \theta(t),
$$

where $\theta \in C_{c}^{\infty}(0, T)$ such that $\theta \geq 0$, and $\xi$ is defined as follows. Let $\left\{\varphi_{j}\right\}_{0 \leq j \leq J}$ be a partition of unity subordinate to open sets $B_{0}, B_{1}, \cdots, B_{J}$ such that $\bar{\Omega} \subset \cup_{j=0}^{J} B_{j}$, $B_{0} \subset \subset \Omega$ and $\partial \Omega \subset \cup_{j=1}^{J} B_{j}$. Let $\hat{\varphi}_{j} \in C_{c}^{\infty}\left(\mathbf{R}^{d}\right), 0 \leq \hat{\varphi}_{j} \leq 1$, such that $\operatorname{supp}\left(\hat{\varphi}_{j}\right) \subset$ $B_{j}$ and $\hat{\varphi}_{j}(x)=1$ on the support of $\varphi_{j}$ so that $\varphi_{j}(x) \hat{\varphi}_{j}(x)=\varphi_{j}(x)$. We use $\varphi_{j}$ as a function of $y$ and $\hat{\varphi}_{j}$ as a function of $x$, and denote $\hat{\varphi}_{j}(x) \varphi_{j}(y)=\psi_{j}(x, y)$. Define

$$
\xi(x, t, y, s)=\sum_{j=0}^{J} \omega_{l}(t-s) \omega_{m}\left(x^{\prime}-y^{\prime}\right) \omega_{n}\left(x_{d}-y_{d}\right) \psi_{j}(x, y),
$$

where $\omega_{l}, \omega_{n}$ are sequences of symmetric mollifiers in $\mathbf{R}, \omega_{m}$ is a sequence of symmetric mollifier in $\mathbf{R}^{d-1}$, and for $j=1,2, \cdots, J, x=\left(x^{\prime}, x_{d}\right), y=\left(y^{\prime}, y_{d}\right)$ are local coordinates induced by $\psi_{j}(x, y)$ in $B_{j}$, that is, $B_{j} \cap \partial \Omega=\left\{x \in B_{j}: x_{d}=\right.$ $\left.\rho_{j}\left(x^{\prime}\right)\right\}, B \cap \Omega=\left\{x \in B_{j}: x_{d}<\rho_{j}\left(x^{\prime}\right)\right\}$ for some Lipschitz continuous function $\rho_{j}: \mathbf{R}^{d-1} \rightarrow \mathbf{R}$.

By taking limit $\delta, \eta \rightarrow 0$ in the entropy error identity (3.8), we obtain the following entropy error inequality.

Theorem 3.5. Let $\theta$ and $\xi$ be defined in Definition 3.4. Then we have the following entropy error inequality

$$
\begin{aligned}
& -\int_{Q \times Q} U_{\varepsilon}\left(u, U_{h}\right) \xi \theta_{t}-\int_{Q \times Q} K_{\varepsilon}\left(u, U_{h}\right) \cdot\left(\nabla_{x} \xi+\nabla_{y} \xi\right) \theta \\
& +\int_{Q \times Q} H_{\varepsilon}^{\prime}\left(A(u)-A\left(U_{h}\right)\right)\left|\nabla_{x} A\left(U_{h}\right)-\nabla_{y} A(u)\right|^{2} \xi \theta \\
\leq & \left.-\int_{Q \times Q} \partial_{t}\left[U_{\varepsilon}\left(U_{h}, u\right)-U_{\varepsilon}\left(u, U_{h}\right)\right)\right] \xi \theta \\
& \left.-\int_{Q \times Q} \nabla_{x}\left[F_{\varepsilon}\left(u, U_{h}\right)-F_{\varepsilon}\left(u, U_{h}\right)\right)\right] \xi \theta \\
& -\int_{Q_{(y, s)}} \int_{\Sigma_{(x, t)}}\left(F_{\varepsilon}\left(u, U_{h}\right)-H_{\varepsilon}\left(A(u)-A\left(U_{h}\right)\right) \nabla_{y} A(u)\right) \cdot \nu_{x} \xi \theta \\
& -\int_{Q_{(x, t)}} \int_{\Sigma_{(y, s)}}\left(F_{\varepsilon}\left(u, U_{h}\right)-H_{\varepsilon}\left(A\left(U_{h}\right)-A(u)\right) \nabla_{x} A\left(U_{h}\right)\right) \cdot \nu_{y} \xi \theta \\
& -\int_{Q_{(y, s)}} \int_{0}^{T}\left\langle\mathcal{R}, H_{\varepsilon}\left(A\left(U_{h}\right)-A(u)\right) \xi \theta\right\rangle d t
\end{aligned}
$$

where $K_{\varepsilon}\left(u, U_{h}\right)=F_{\varepsilon}\left(u, U_{h}\right)-H_{\varepsilon}\left(A(u)-A\left(U_{h}\right)\right)\left(\nabla_{y} A(u)-\nabla_{x} A\left(U_{h}\right)\right), \Sigma=\partial \Omega \times$ $(0, T)$, and $\Sigma_{(x, t)}$ or $\Sigma_{(y, s)}$ are the domain of integration of $\Sigma$ with respect to $(x, t)$ or $(y, s)$ respectively. 
For any $\varepsilon>0$ and $z \in \mathbf{R}$, define

$$
\nu(\varepsilon, z)=\min \left\{A^{\prime}(s):|A(s)-A(z)| \leq \varepsilon\right\} .
$$

Assume $A^{\prime} \circ A^{-1}$ is Lipschitz, then we have the following elementary estimate which extends the result in [18, Corollary 6.4]

$$
\left|\partial_{z}\left[U_{\varepsilon}(z, k)-U_{\varepsilon}(k, z)\right]\right| \leq \frac{\varepsilon}{\nu(\varepsilon, z)} \mathcal{K}_{1}, \quad\left|\partial_{z}\left[F_{\varepsilon}(z, k)-F_{\varepsilon}(k, z)\right]\right| \leq \frac{\varepsilon}{\nu(\varepsilon, z)} \mathcal{K}_{2},(3
$$

where $k, z \in \mathbf{R}, \mathcal{K}_{1}=\mathrm{L}\left(A^{\prime} \circ A^{-1}\right), \mathcal{K}_{2}=\mathcal{K}_{1}\left\|f^{\prime}\right\|_{L^{\infty}(\mathbf{R})}+\mathrm{L}\left(f^{\prime}\right)$ with $\mathrm{L}\left(A^{\prime} \circ A^{-1}\right)$ and $\mathrm{L}\left(f^{\prime}\right)$ being the Lipschitz constant of $A^{\prime} \circ A^{-1}$ and $f^{\prime}$ respectively.

To complete the Kružkov "doubling of variables" technique, we let first $l, m \rightarrow$ $\infty$ then $n \rightarrow \infty$ in the entropy error inequality (3.9). The first two terms on the right-hand side of (3.9) can be treated by using (3.10) and the third and fourth terms can be shown to tend to zero. Thus we have

$$
\begin{gathered}
-\int_{Q} U_{\varepsilon}\left(u, U_{h}\right) \theta_{t}+\int_{Q} H_{\varepsilon}^{\prime}\left(A(u)-A\left(U_{h}\right)\right)\left|\nabla\left(A\left(U_{h}\right)-A(u)\right)\right|^{2} \theta \\
\leq \mathcal{K} \varepsilon \int_{Q} \frac{1}{\nu\left(\varepsilon, U_{h}\right)}\left(\left|\partial_{t} U_{h}\right|+\left|\nabla_{x} U_{h}\right|\right) \theta-\int_{0}^{T}\left\langle\mathcal{R}, H_{\varepsilon}\left(A\left(U_{h}\right)-A(u)\right) \theta\right\rangle d t .
\end{gathered}
$$

where $\mathcal{K}=\max \left(\mathcal{K}_{1}, \mathcal{K}_{2}\right)$.

To proceed, we introduce the interior residual

$$
R^{n}:=\bar{g}^{n}-\frac{U_{h}^{n}-\bar{U}_{h}^{n-1}}{\tau_{n}}+\Delta A\left(U_{h}^{n}\right) \quad \text { on any } K \in \mathcal{M}^{n}
$$

where we recall that $\bar{g}^{n}=\tau_{n}^{-1} \int_{t^{n-1}}^{t^{n}} g(x, t) d t$.

Theorem 3.6. Let $\varepsilon_{0}=\sum_{i=1}^{3} \mathcal{E}_{i}$, where $\mathcal{E}_{1}, \mathcal{E}_{2}, \mathcal{E}_{3}$ are the error indicators defined below. For any $m \geq 1$, let $Q_{m}=\Omega \times\left(0, t^{m}\right)$, and define

$$
\Lambda_{m}=\max \left(1, \int_{Q_{m}} \frac{1}{\nu\left(\varepsilon_{0}, U_{h}\right)}\left(\left|\partial_{t} U_{h}\right|+\left|\nabla U_{h}\right|\right)+\int_{\Omega} \frac{1}{\nu\left(\varepsilon_{0}, U_{h}^{m}\right)}\right)
$$

where for any $z \in \mathbf{R}, \nu\left(\varepsilon_{0}, z\right)=\min \left\{A^{\prime}(s):|A(s)-A(z)| \leq \varepsilon_{0}\right\}$. Then there exists a constant $C$ depending only on the minimum angles of the meshes $\mathcal{M}^{n}$, $n=1, \cdots, m$, such that the following a posteriori error estimate is valid

$$
\left\|u^{m}-U_{h}^{m}\right\|_{L^{1}(\Omega)} \leq \mathcal{E}_{0}+\mathcal{E}_{4}+\mathcal{E}_{5}+C \Lambda_{m}^{1 / 2}\left(\sum_{i=1}^{3} \mathcal{E}_{i}\right),
$$


where the error indicators $\mathcal{E}_{i}, i=0, \cdots, 5$, are defined by

$$
\begin{array}{lll}
\mathcal{E}_{0} & =\left\|u_{0}-U_{h}^{0}\right\|_{L^{1}(\Omega)} & \text { initial error } \\
\mathcal{E}_{1} & =\left(\sum_{n=1}^{m} \tau_{n}\left\|h_{n}^{1 / 2} \llbracket \nabla A\left(U_{h}^{n}\right) \rrbracket\right\|_{L^{2}(\Omega)}^{2}\right)^{1 / 2} & \text { jump residual } \\
\mathcal{E}_{2}=\left(\sum_{n=1}^{m} \tau_{n}\left\|h_{n} R^{n}\right\|_{L^{2}(\Omega)}^{2}\right)^{1 / 2} & \text { interior residual } \\
\mathcal{E}_{3}=\left(\sum_{n=1}^{m} \tau_{n}\left\|\nabla\left(A\left(U_{h}^{n}\right)-A\left(U_{h}^{n-1}\right)\right)\right\|_{L^{2}(\Omega)}^{2}\right)^{1 / 2} & \text { time residual } \\
\mathcal{E}_{4}=\sum_{n=1}^{m} \int_{t^{n-1}}^{t^{n}}\left\|\frac{U_{h}^{n}-\bar{U}_{h}^{n-1}}{\tau_{n}}-\left(\partial_{t} U_{h}+\operatorname{div} f\left(U_{h}\right)\right)\right\|_{L^{1}(\Omega)} d t & \text { characteristic } \\
\mathcal{E}_{5} & =\sum_{n=1}^{m} \int_{t^{n-1}}^{t^{n}}\left\|g-\bar{g}^{n}\right\|_{L^{1}(\Omega)} d t & \text { and coarsening }
\end{array}
$$

In the case of strong diffusion $A^{\prime}(s) \geq \beta>0$ for any $s \in \mathbf{R}$ and $A^{\prime}$ is uniformly Lipschitz continuous, then $\Lambda_{n}$ is bounded by $\beta^{-1}\left\|U_{h}\right\|_{B V\left(Q_{n}\right)}$ which is expected to be bounded in practical computations. The a posteriori error estimator in Theorem 3.6 then recovers the standard a posteriori error estimator derived in the literature for parabolic problems $[34,9]$. In particular, the space error indicators $\mathcal{E}_{1}^{n}, \mathcal{E}_{2}^{n}$, which control the adaptation of finite element meshes at each time step, are sharp in the sense that a local lower bound for the error can be established by extending the argument in [9, Theorem 2.2] for linear parabolic equations.

We also remark that the method of the a posteriori error analysis here is different from those for nonlinear conservation laws in $[17,18,27]$ or nonlinear degenerate parabolic equations in [32]. Recall that there are several parameters introduced in the analysis

- The regularizing parameter $\varepsilon$ in $H_{\varepsilon}(z)$;

- The boundary layer sequence parameters $\delta, \eta$ and the mollifier parameters $l, m, n$.

The analysis for Cauchy problems in $[17,18,27]$ is based on letting $\varepsilon \rightarrow 0$ and taking finite mollifier parameters $l, m, n$. The analysis in [32] takes both finite $\varepsilon$ and finite mollifier parameters $l, m, n$. Note that there are no boundary layer sequence parameters $\delta, \eta$ for the analysis for Cauchy problems. The analysis in this paper is based on letting $\delta, \eta \rightarrow 0$ and $l, m, n \rightarrow \infty$ but taking a finite $\varepsilon$. We are not able to use the same technique as that in $[17,18,27,32]$ by choosing finite mollifier parameters $l, m, n$ to treat the problem with boundary conditions.

Based on the a posteriori error estimate in Theorem 3.6, an adaptive algorithm is proposed and implemented in [11]. In particular, the numerical experiments in [11] indicate that the total estimated error is roughly proportional to $M^{-1 / 3}$, i.e. 
$\eta \approx C M^{-1 / 3}$ for some constant $C>0$. This implies the quasi-optimal decay of the error

$\left\|u-U_{h}\right\|_{L^{\infty}\left(0, T ; L^{1}(\Omega)\right)}+\int_{Q} H_{\varepsilon}^{\prime}\left(A(u)-A\left(U_{h}\right)\right)\left|\nabla\left(A\left(U_{h}\right)-A(u)\right)\right|^{2} \leq C M^{-1 / 3}$

is valid asymptotically. Here $M$ is the sume of the number of elements of the space meshes over all time steps.

Figure 3.1 shows the meshes and the surface plots of the solutions at time $t=0.251278$ and $t=0.500878$ for the Burger's equation with small viscosity

$$
\frac{\partial u}{\partial t}+u \partial_{x} u-\varepsilon \Delta u=0 \quad \text { in } Q
$$

where $\Omega=(0,1)^{2}, T=1.0, \varepsilon=10^{-3}$, and the initial condition and boundary condition

$$
\left.u(x, y, t)\right|_{\partial \Omega}=u_{0}(x, y)=0.5 \sin (\pi x)+\sin (2 \pi x) .
$$

The adaptive algorithm is based on the a posteriori error estimate in Theorem 3.6 and is described in [11]. We observe from Figure 3.1 that the method captures the internal and boundary layers of the solution.

Acknowledgment. The author would like to thank Shibin Dai, Guanghua Ji, Feng Jia, Xuezhe Liu, Ricardo H. Nochetto, Alfred Schmidt, and Haijun Wu for the joint work through the years.

\section{References}

[1] Alt, H.W. and Luckhaus, S., Quasilinear elliptic-parabolic differential equations, Math. Z. 183 (1983), 311-341.

[2] Babuška, I. and Miller, A., A feedback finite element method with a posteriori error estimation: Part I. The finite element method and some basic properties of the a posteriori error estimator, Comput. Meth. Appl. Mech. Engrg. 61 (1987), 1-40.

[3] Babuška, I. and Rheinboldt, C., Error estimates for adaptive finite element computations, SIAM J. Numer. Anal. 15 (1978), 736-754.

[4] Berenger, J.-P., A perfectly matched layer for the absorption of electromagnetic waves. J. Comput. Physics 114 (1994), 185-200.

[5] Binev, P., Dahmen, W. and DeVore, R., Adaptive finite element methods with convergence rates, Numer. Math. 97 (2004), 219-268.

[6] Carrillo, J., Entropy solutions for nonlinear degenerate problems, Arch. Rational Mech. Anal. 147 (1999), 269-361.

[7] Chen, Z. and Dai, S., Adaptive Galerkin methods with error control for a dynamical Ginzburg-Landau model in superconductivity, SIAM J. Numer. Anal. 38 (2001), 1961-1985.

[8] Chen, Z. and Dai, S., On the efficiency of adaptive finite element methods for elliptic problems with discontinuous coefficients. SIAM J. Sci. Comput. 24 (2002), 443-462. 
[9] Chen, Z. and Jia, F., An adaptive finite element method with reliable and efficient error control for linear parabolic problems, Math. Comp. 73 (2004), 1163-1197.

[10] Chen, Z. and Ji, G., Adaptive computation for convection dominated diffusion problems, Science in China, 47 Supplement (2004), 22-31.

[11] Chen, Z. and Ji, G., Sharp $L^{1}$ a posteriori error analysis for nonlinear convectiondiffusion problems, Math. Comp. (to appear).

[12] Chen, Z. and Liu, X., An Adaptive Perfectly Matched Layer Technique for Timeharmonic Scattering Problems, SIAM J. Numer. Anal. (to appear).

[13] Chen, Z. and Nochetto, R.H., Residual type a posteriori error estimates for elliptic obstacke problems, Numer. Math. 84 (2000), 527-548.

[14] Chen, Z., Nochetto, R.H., and Schmidt, A., A characteristic Galerkin method with adaptive error control for continuous casting problem, Comput. Methods Appl. Mech. Engrg. 189 (2000), 249-276.

[15] Chen, Z., Nochetto, R.H., and Schmidt, A., Error control and adaptivity for a phase relaxation model, Math. Model. Numer. Anal. 34 (2000), 775-797.

[16] Chen, Z. and Wu, H., An adaptive finite element method with perfectly matched absorbing layers for the wave scattering by periodic structures. SIAM J. Numer. Anal. 41, (2003), 799-826.

[17] Cockburn, B., Coquel, B.F. and Lefloch, P.G., An error estimate for finite volume methods for multidimensional conservation laws, Math. Comp. 63 (1994), 77-103.

[18] Cockburn, B. and Gremaud, P.-A., Error estimates for finite element methods for scalar conservation laws, SIAM J. Numer. Anal. 33 (1996), 522-554.

[19] Collino, F. and Monk, P.B., The perfectly matched layer in curvilinear coordinates. SIAM J. Sci. Comput. 19 (1998), 2061-2090.

[20] Colton D. and Kress R., Integral Equation Methods in Scattering Theory. John Wiley \& Sons, New York, 1983.

[21] Hohage, T., Schmidt, F. and Zschiedrich, L., Solving time-harmonic scattering problems based on the pole condition. II: Convergence of the PML method. SIAM J. Math. Anal., (to appear).

[22] Lassas, M. and Somersalo, E., On the existence and convergence of the solution of PML equations. Computing 60 (1998), 229-241.

[23] Dörfler, W., A convergent adaptive algorithm for Possion's equations, SIAM J. Numer. Anal. 33 (1996), 1106-1124.

[24] Douglas Jr., J. and Russell, T.F., Numerical methods for convection-dominated diffusion problem based on combining the method of characteristic with finite element or finite difference procedures, SIAM J. Numer. Anal. 19 (1982), pp. 871-885.

[25] Eriksson, K. and Johnson, C., Adaptive finite element methods for parabolic problems I: A linear model problem, SIAM J. Numer. Anal. 28 (1991), 43-77.

[26] Houston, P. and Süli, E., Adaptive Lagrange-Galerkin methods for unsteady convection-diffusion problems, Math. Comp. 70 (2000), 77-106.

[27] Kröner, D. and Ohlberger, M., A posteriori error estimates for upwind finite volume schemes for nonlinear conservation laws in multi-dimensions, Math. Comp. 69 (2000), 25-39. 
[28] Kružkov, N.N., First order quasi-linear equations in several independent variables, Math. USSR Sbornik 10 (1970), 217-243.

[29] Mascia, C., Porretta, A. and Terracina, A., Nonhomogeneous Dirichlet problems for degenerate parabolic-hyperbolic equations, Arch. Rational Mech. Anal. 163 (2002), $87-124$.

[30] Morin, P., Nochetto. R.H. and Siebert, K.G., Data oscillation and convergence of adaptive FEM, SIAM J. Numer. Anal. 38 (2000), 466-488.

[31] Nochetto, R.H., Schmidt, A. and Verdi, C., A posteriori error estimation and adaptivity for degenerate parabolic problems, Math. Comp. 69 (2000), 1-24.

[32] Ohlberger, M., A posteriori error estimates for vertex centered finite volume approximations of convection-diffusion-reaction equations, Math. Model. Numer. Anal. 35 (2001), 355-387.

[33] Otto, F., $L^{1}$-contraction and uniqueness for quasilinear elliptic-parabolic equations, J. Diff. Equations 131 (1996), 20-38.

[34] Picasso, M., Adaptive finite elements for a linear parabolic problem, Comput. Methods Appl. Mech. Engrg. 167 (1998), 223-237.

[35] Pironneau, O., On the transport-diffusion algorithm and its application to the Navier-Stokes equations, Numer. Math. 38 (1982), pp. 309-332.

[36] Schmidt, A. and Siebert, K.G., ALBERT: An adaptive hierarchical finite element toolbox, IAM, University of Freiburg, 2000. http://www.mathematik.unifreiburg.de/IAM/Research/projectsdz/albert.

[37] Teixeira, F.L. and Chew, W.C., Advances in the theory of perfectly matched layers. In Fast and Efficient Algorithms in Computational Electromagnetics (ed. by W.C. Chew) Artech House, Boston, 2001, 283-346.

[38] Turkel, E. and Yefet, A., Absorbing PML boundary layers for wave-like equations. Appl. Numer. Math. 27 (1998), 533-557.

[39] Verfürth, R., A Review of A Posteriori Error Estimation and adaptive Mesh Refinement Techniques, Teubner (1996).

LSEC, Institute of Computational Mathematics, Academy of Mathematics and Systems Science, Chinese Academy of Sciences, Beijing 100080, China.

E-mail: zmchen@lsec.cc.ac.cn 

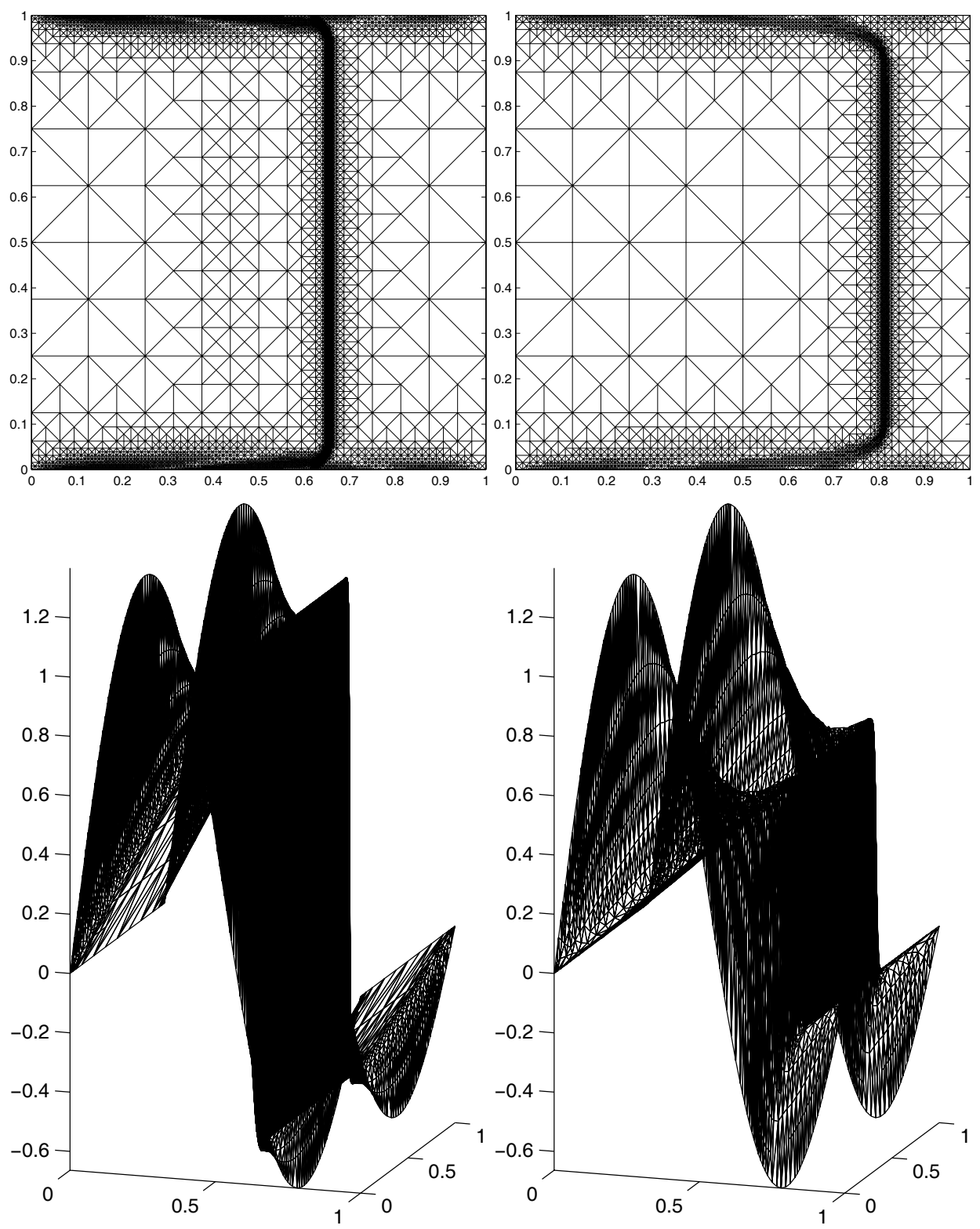

Figure 3.1. The meshes (top) and the surface plots (bottom) of the solutions $t=0.400317$ (left) and $t=1.0$ (right) with 35286 and 5020 nodes. 